Esther Klann, Eric Todd Quinto, Ronny Ramlau

\title{
Wavelet methods for a weighted sparsity penalty for region of interest tomography
}

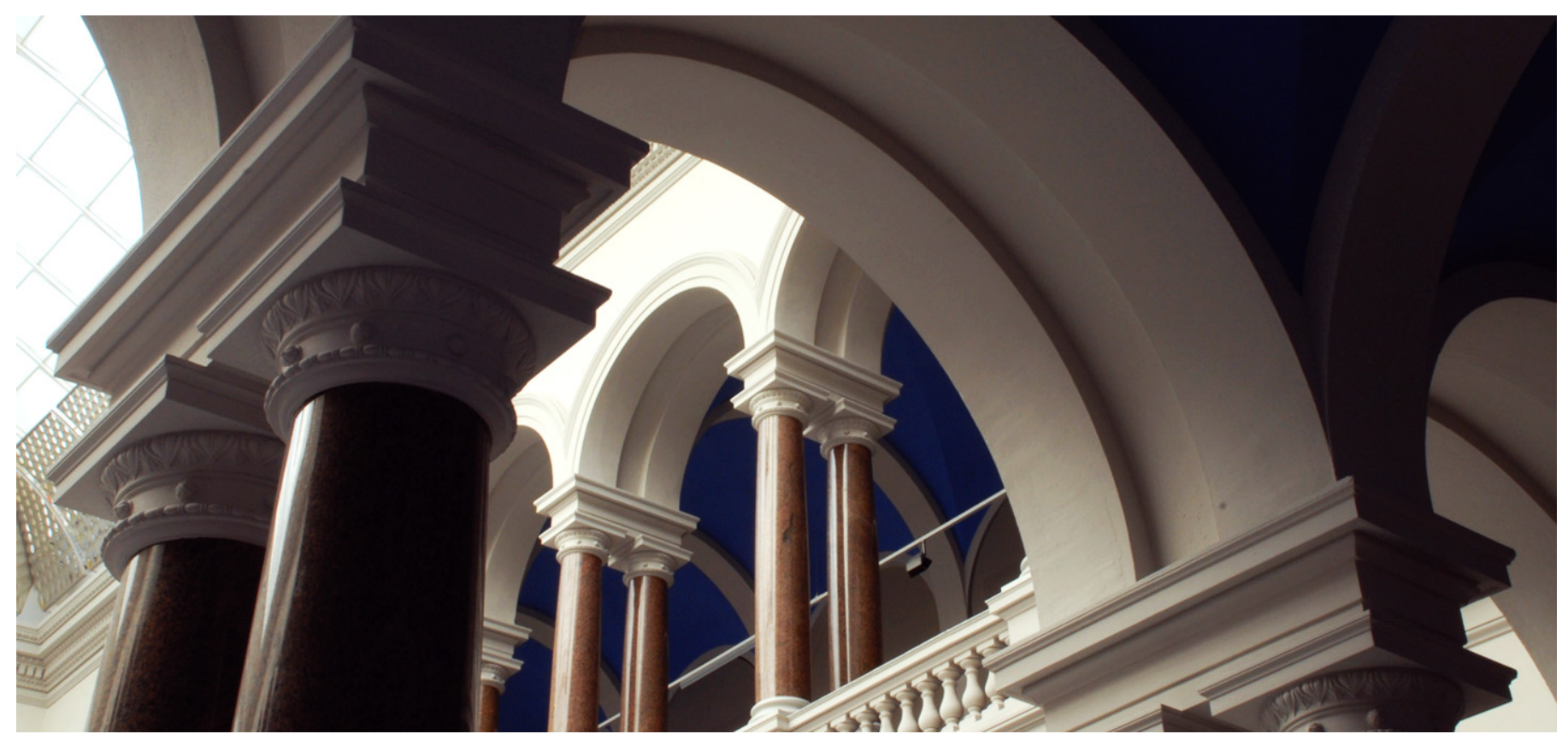

\section{Suggested Citation}

Esther Klann et al: Wavelet methods for a weighted sparsity penalty for region of interest tomography.b Inverse Problems 31 (2015) 025001. DOI: 10.1088/0266-5611/31/2/025001

This is an author-created, un-copyedited version of an article accepted for publication/published in Inverse problems. IOP Publishing Ltd is not responsible for any errors or omissions in this version of the manuscript or any version derived from it. The Version of Record is available online at https://doi.org/10.1088/0266-5611/31/2/025001. 


\title{
WAVELET METHODS FOR A WEIGHTED SPARSITY PENALTY FOR REGION OF INTEREST TOMOGRAPHY
}

\author{
ESTHER KLANN , ERIC TODD QUINTO , AND RONNY RAMLAU
}

\begin{abstract}
We consider region of interest (ROI) tomography of piecewise constant functions. We prove that continuous ROI data determine piecewise constant functions. Additionally, an algorithm is developed for ROI tomography of piecewise constant functions using a Haar wavelet basis. A weighted $\ell_{p}$-penalty is used with weights that depend on the relative location of wavelets to the region of interest. We prove that the proposed method is a regularization method, i.e., that the regularized solutions converge to the exact piecewise constant solution if the noise tends to zero. Tests on phantoms demonstrate the effectiveness of the method.
\end{abstract}

\section{INTRODUCTION}

In this article, we consider the interior problem or region of interest (ROI) tomography; tomographic data are given only over lines meeting a region of interest inside the body, and the goal is to image that region. This comes up in micro-CT in which researchers are interested in only a small part of an object and in medical CT in which doctors want to limit X-ray dose and know the location of what they want to image $[21,22,62]$. If $\Omega$ is the region of interest, then ROI data of a function $f$ are the integrals $R f(\ell)$ for all lines $\ell$ that intersect $\Omega$. The goal is to image $f$ in $\Omega$ from this data.

However, in general, $f$ is not determined from ROI data (see Example 2.1). One can recover singularities of $f$ in the ROI from ROI data. This is true because $R$ is an elliptic Fourier integral operator and $R^{*} R$ is an elliptic pseudodifferential operator (e.g., [28, 49]). This fact is one motivation for Lambda tomography [22,62], a local reconstruction method from ROI data that reconstructs singularities of objects and clearly shows boundaries of parts of the object.

In this article, we consider the ROI problem for piecewise constant (PC) functions. Any $\mathrm{PC}$ function can be written as the finite linear combination of characteristic functions:

$$
f(x)=\sum_{n=1}^{N} a_{n} \chi_{\Omega_{n}}(x) .
$$

We assume that each set $\Omega_{n}$ is compact with piecewise smooth boundary and nontrivial interior and $\chi_{\Omega_{n}}$ denotes the characteristic function of $\Omega_{n}$. Note that we only allow a finite number of sets, which appears realistic for many applications.

The authors thank the generosity of RICAM and Tufts University for providing support for this research. The first author was supported by FWF grant T 529-N18. The second author was supported in part by NSF Grant DMS 1311558. The third author was supported partly by Doctoral Program "Computational Mathematics." The authors would like to thank Jan Boman for astute comments about Section 2, including conjecturing Theorem 2.2 for piecewise polynomial functions. The authors thank both referees for valuable comments that made the article clearer and for calling our attention to [67]. 
We prove that PC functions are determined by their ROI data (Theorem 2.2), and we present a new sparsity promoting reconstruction method for ROI CT. We propose a weighted wavelet reconstruction scheme where the weights are chosen according to the localization of the wavelet relative to the ROI. Due to our uniqueness result for PC functions (Theorem 2.2), we have convergence of the reconstruction method to that unique solution.

The mathematical model for X-raying an object is the Radon transform of the body density $f[47]$,

$$
R f(\omega, s)=\int_{\mathbb{R}} f\left(s \omega+t \omega^{\perp}\right) d t
$$

$(\omega, s) \in S^{1} \times \mathbb{R}$ and $\omega^{\perp} \in \mathbb{R}^{2}$ is a vector orthogonal to $\omega$. Here $S^{1}$ denotes the unit sphere in $\mathbb{R}^{2}$, i.e., the set of all orientations in two-dimensional space. The pair $(\omega, s) \in S^{1} \times \mathbb{R}$ defines a line $\ell=\ell(\omega, s) \in \mathbb{R}^{2}$ perpendicular to $\omega$ at directed distance $s$ to the origin. For convenience, we often denote the argument of the Radon transform by $\ell$, i.e., we use $R f(\ell)$ for the Radon transform along the line $\ell$.

Assuming the function $f$ to be supported on some set $D$, in ROI tomography, data are given only on lines intersecting some subset $\Omega$ (the ROI) of $D$. To indicate this data limitation, we define the operator $R_{\text {lim }}$ with

$$
R_{\lim } f(\omega, s):= \begin{cases}R f(\omega, s) & \text { if } \ell(\omega, s) \cap \Omega \neq \emptyset \\ 0 & \text { else. }\end{cases}
$$

To solve the ROI tomography problem, the equation $R_{\lim } f=z_{\lim }$ has to be inverted. Already the full data problem of solving $R f=z$ in $L_{2}$ is ill-posed [20,42], and regularization methods have to be applied.

The goal of this article is to compute a regularized solution to the problem of solving $R_{\lim } f=z_{\lim }$ from possible noisy data $z_{\lim }^{\delta}$ by minimizing a functional of the form

$$
\left\|R_{\lim } f-z_{\lim }^{\delta}\right\|_{L_{2}\left(S^{1} \times \mathbb{R}\right)}^{2}+\alpha\|F f\|_{p, \omega}^{p} .
$$

The term $\|\cdot\|_{p, \omega}^{p}$ denotes a weighted $\ell_{p}$-norm with strictly positive weights $0<C \leq \omega_{n}$ and the operator $F$ maps $f$ to its Fourier coefficients with respect to a basis or frame, $F f:=\left(\left\langle f, \varphi_{n}\right\rangle\right)_{n=1, \ldots}$. For $p<2$, such an approach is known to promote sparsity. In most applications the weights are either constant or given as a fixed sequence.

In contrast, we use a set of weights that influence the reconstruction according to the location of the basis (or frame) function in relation to the region of interest. The minimization of the functional will be done with respect to the space of piecewise constant functions that can be represented as a finite sum of Haar (db1) wavelets. The weights are chosen such that wavelets that are contained within the ROI, or contain the ROI, contribute essentially to the reconstruction within the ROI; whereas wavelets located far away from the ROI or with only a small part inside the ROI have a smaller influence on the reconstruction quality inside the ROI. By treating these classes of wavelets differently through the weights we aim at a sparse and stable reconstruction method that emphasizes high quality inside the ROI but still allows features to be reconstructed outside.

We now give a short overview of the literature on limited data tomography, especially region of interest tomography, wavelet-based methods in tomography, and inverse problems with sparsity constraints. There are some approaches to pseudolocal tomography using almost completely local data; in $[16,17,58]$ an additional coarse angular sampling of the 
whole domain is assumed whereas in [56] an additional margin to the region of interest is used. Total variation approaches to the interior problem are studied in $[64,66]$. Authors have shown that wavelet based local tomography allows one to find density differences and to put Lambda tomography and pseudolocal tomography [35] in a wavelet context [46]. Waveletbased approaches for limited angle tomography can be found, e.g., in [55,57]. In [34], an adaptive regularization based on frequency components is applied to limited tomography with additional coarse angular sampling. For sparse tomography, i.e., full angle of view but only few directions, we refer to [29] for a wavelet-based sparsity promoting approach. Inverse problems using wavelets and/or sparsity constraints are discussed in $[1-9,14,15,18,19,23-$ $27,32,33,38,40,41,50-54,60,68]$.

The closest articles to our work are [30,66,67] and [48]. Yu and Wang [66] and Han, Yu and Wang [30] have developed a total variation reconstruction method for the ROI problem when the ROI is a disk, $D$, based on the fact that a PC function, $f$, has a lower total variation (semi)norm in $D$ than any other solution, $h$, to the continuous problem $R h(\ell)=R f(\ell)$ for all lines intersecting $D$. Niinimäki et al. [48] have proposed a Bayesian multiresolution method for local tomography reconstruction in dental x-ray imaging. They use a wavelet basis for the representation of the dental structures with a high resolution inside the ROI and coarser resolution outside the ROI. Their approach is closely related to our proposed method. However, whereas their method does not allow any fine details outside the ROI, our method will reconstruct significant fine details outside the ROI as shown in the numerics section.

The article is organized as follows. In Section 2 we prove that PC functions are uniquely determined from region of interest data. In Section 3 we present the general setup and in Section 4 we describe the used regularization method. In Section 5 we discuss the choice of the weights and in Section 6 we present numerical examples. Section 7 is the conclusion.

\section{UNIQUENESS AND NONUNIQUENESS FOR THE INTERIOR PROBLEM}

In this section, we examine theoretical results about uniqueness for ROI CT for PC functions, which we will use in Section 4.

We first provide an example to show that arbitrary functions are not determined by ROI data (Ex. 2.1), even in the ROI, without some a priori information. Next, we prove the surprising result, Thm. 2.2, that PC functions are determined everywhere from ROI data.

Throughout this section, we use the notation $D(r)$ for the open disk centered at the origin in $\mathbb{R}^{2}$ and with radius $r$.

Example 2.1. Let $\varepsilon$ and $L$ be chosen so $0<\varepsilon<L$. We construct a null function $f$ supported in $D(L)$ for the ROI problem where $R f=0$ for lines meeting $D(\varepsilon)$ but $f$ is not identically zero in $D(\varepsilon)$.

Let $g(s)$ be a smooth, nonnegative function with support the interval $[\varepsilon, L]$. We will view $g$ as a function of $(s, \omega)$ that is constant in $\omega$.

The Radon transform has a simple inversion formula for radial functions [11, p. 2725, equation (18)]. So, if $R f=g$, then

$$
f(r)=-\frac{1}{\pi} \frac{d}{d r} \int_{r}^{L} \underset{3}{\frac{r}{s}}\left(s^{2}-r^{2}\right)^{-1 / 2} g(s) d s .
$$


Therefore, for $|r|<\varepsilon$,

$$
f(r)=-\frac{1}{\pi} \frac{d}{d r} \int_{\varepsilon}^{L} \frac{r}{s}\left(s^{2}-r^{2}\right)^{-1 / 2} g(s) d s=-\frac{1}{\pi} \int_{\varepsilon}^{L}\left(s^{2}-r^{2}\right)^{-3 / 2}(s g(s)) d s,
$$

where we have used the support restriction on $g$ to specify the limits of integration. Now it is clear that $f(0)$ is strictly negative and so $f$ is a null function that is nonzero in the ROI.

Peter Maaß constructs a null function for ROI data in [45] and Natterer shows that null functions are fairly constant in the ROI [47, p. 169, VI.4].

Several uniqueness results are valid for ROI data. First, if one knows $f$ on a subset of the region of interest, then one can determine $f$ in the ROI using ROI data. Constructive proofs and inversion methods have been given in $[12,65]$ by analytically continuing a Hilbert transform, and Corollary 2.5 provides a proof that $f$ is unique everywhere. In Remark 2.6, we discuss results in $[64,67]$ that are closely related to the theorems in this section.

As noted in the introduction, our goal is to reconstruct PC functions (as in equation (1.1)). Our next theorem shows that PC functions are determined everywhere from ROI data. This is in contrast to the general case in which there is a large null space, as shown in Example 2.1.

Theorem 2.2. Let $\Omega$ be a bounded open set and let $f$ be a compactly supported integrable $\left(L^{1}\right)$ function that is $P C$ in $\Omega$. Then, data $R f(\ell)$ for lines $\ell$ intersecting $\Omega$ determine $f$ everywhere. That is, if $R f_{1}(\ell)=R f_{2}(\ell)$ for all lines $\ell$ intersecting $\Omega$ and if $f_{1}$ and $f_{2}$ are $P W C$ in $\Omega$, then $f_{1}=f_{2}$ everywhere.

Note that the function $f$ in the theorem can be supported outside $\Omega$, and $f$ is not necessarily $\mathrm{PC}$ outside of $\Omega$. Therefore, this theorem applies to more general functions than those that are PC everywhere. Corollary 2.5 is a stronger uniqueness result that follows from our proof.

Proof of Theorem 2.2. We make two reductions. First, let $f$ be a function of compact support that is $\mathrm{PC}$ in $\Omega$ and assume $R f(\ell)=0$ for lines $\ell$ intersecting $\Omega$. We will prove that $f=0$ everywhere. By linearity, this will prove the theorem.

Second, since $\Omega$ is open and $f$ is $\mathrm{PC}$ in $\Omega$, we know $\Omega$ contains an open disk on which $f$ is constant. By translating the center of the disk to the origin and using that the Radon transform is translation invariant, we may assume the following:

Hypothesis 2.3. $f$ is an integrable function of compact support that is constant in the disk $D(\varepsilon)$ for some $\varepsilon>0$, and $R f$ is zero for all lines intersecting $D(\varepsilon)$.

Under this hypothesis, we will show $f=0$ everywhere, and this will prove the theorem.

Lemma 2.4. Let $f$ satisfy Hyp. 2.3. Then, the circular means (or averages) of $f$ over all circles with centers in $D(\varepsilon)$ are zero.

Proof of Lemma 2.4. By a standard convolution argument, one may assume $f$ is a smooth function of compact support. Let $\varphi$ be a smooth radial function supported in $D(1)$ with integral 1 and let $\varphi_{n}(x)=n^{2} \varphi(n x)$. Then $f * \varphi_{n} \rightarrow f$ in $L^{1}$. Because $R\left(f * \varphi_{n}\right)=R f *_{p} R \varphi_{n}$ and $R \varphi_{n}$ is supported in $[0,2 \pi] \times[-1 / n, 1 / n]$, one sees for $n>1 / \varepsilon$ that $f * \varphi_{n}$ satisfies Hypothesis 2.3 on $D(\varepsilon-1 / n)$ and is smooth. Finally, once we have proven that $f * \varphi_{n}=0$, then, by taking the limit as $n \rightarrow \infty$, we see $f=0$. (This same reduction works to prove 
Lemma 2.4 for $f$ a distribution of compact support, and then this lemma can be used to prove Theorem 2.2 for distributions of compact support that are PC functions in the ROI.)

Therefore, we may assume that $f$ is smooth and has compact support and is constant in $D(\varepsilon)$.

To show that circular means (or average) of $f$ over all circles centered at the origin are zero, we consider $0^{\text {th }}$ order angular Fourier coefficient of $f$,

$$
f_{0}(r)=\frac{1}{2 \pi} \int_{\theta \in S^{1}} f(r \theta) d \theta
$$

Note that $f_{0}$ can be defined for all $r \in \mathbb{R}$ and $f_{0}(r)$ is the circular mean of $f$ over the circle of radius $|r|$ centered at the origin. Furthermore, for $|r|<\varepsilon$, this integral is constant since $f$ is constant in $D(\varepsilon)$. Therefore, all derivatives

$$
f_{0}^{(m)}(r)=0 \text { for } m=1,2,3, \ldots \text { and }|r|<\varepsilon .
$$

We now view $f_{0}$ as a radial function on $\mathbb{R}^{2}$. For any $\theta$, let $g=R f_{0}(\theta, s)$. Because $f_{0}$ is radial, $g$ is independent of $\theta$ and can be viewed as a function of $s, g=g(s)$.

First, we show $f_{0}(r)=0$ for all $r$; equivalently, all averages of $f$ over all circles centered at the origin are zero.

Because the Radon transform is rotation invariant, it commutes with averaging over circles centered at the origin (Fubini's Theorem can be used to commute the integrals). This means that

$$
g(s)=R f_{0}(\theta, s)=\frac{1}{2 \pi} \int_{\omega \in S^{1}} R f(\omega, s) d \omega .
$$

Since $R f(\theta, s)=0$ for all lines intersecting $D(\varepsilon)$ (i.e., $|s|<\varepsilon), g(s)=0$ for $|s|<\varepsilon$.

Assume $L>\varepsilon$ is chosen so that $\operatorname{supp}(f) \subset D(L-\varepsilon)$ (this choice will be useful in the second part of the proof). Therefore, the radial function $f_{0}$ is supported in $D(L)$. Applying (2.1) to $R f_{0}=g$, we see for $|r|<\varepsilon$ that

$$
f_{0}(r)=-\frac{1}{\pi} \frac{d}{d r} \int_{\varepsilon}^{L} \frac{r}{s}\left(s^{2}-r^{2}\right)^{-1 / 2} g(s) d s=-\frac{1}{\pi} \int_{\varepsilon}^{L}\left(s^{2}-r^{2}\right)^{-3 / 2}(s g(s)) d s
$$

Taking a derivative in $r$ of the right-hand expression in (2.3), using (2.2), and then dividing by a factor of $r$, we see, for $|r|<\varepsilon$ that

$$
0=\int_{\varepsilon}^{L}\left(s^{2}-r^{2}\right)^{-5 / 2}(s g(s)) d s .
$$

By continuing this pattern-taking a derivative in $r$ then dividing by a factor of $r$, we see that

$$
0=\int_{\varepsilon}^{L}\left(s^{2}-r^{2}\right)^{-(2 n+5) / 2}(s g(s)) d s \text { for } n=0,1,2,3, \ldots \text { and }|r|<\varepsilon .
$$

Now setting $r=0$, we see that

$$
0=\int_{\varepsilon}^{L} s^{-2 n}\left(s^{-4} g(s)\right) d s \text { for } n=0,1,2, \ldots
$$

By the Stone Weierstrass Theorem, the algebra generated by $\left\{s^{-2 n} \mid n=0,1,2, \ldots\right\}$ is dense in $C([\varepsilon, L])$. Therefore, $s^{-4} g(s)=0$ almost everywhere for $s \in[\varepsilon, L]$. Since $g=0$ in $[0, \varepsilon]$, 
we see that $g=0$. Since the Radon transform is injective, $f_{0}(r)=0$ for all $r$. This shows the circular means of $f$ are zero over all circles centered at the origin.

In the second part of the proof, we use the same argument to show that circular means of $f$ are zero over all circles with centers in $D(\varepsilon)$. Let $x \in D(\varepsilon)$ and assume $\varepsilon^{\prime}=\|x-0\|$. Note that $0<\varepsilon^{\prime}<\varepsilon$. Then, one can translate the plane so that $x$ is mapped to zero. Let $\tilde{f}(y)=f(y-x)$, then $\tilde{f}$ is constant on $D\left(\varepsilon-\varepsilon^{\prime}\right)$ and supported in $D(L)$ since $f$ is supported in $D(L-\varepsilon)$. Furthermore integrals of $\tilde{f}$ are zero for all lines meeting $D\left(\varepsilon-\varepsilon^{\prime}\right)$ since $R$ is translation invariant. The same proof as for $x=0$ works except the lower limit of integration is $\left(\varepsilon-\varepsilon^{\prime}\right)$ not $\varepsilon$ in $(2.3),(2.4)$, and $(2.5)$.

We now describe how we use the proof of $[31$, Lemma 2.7, p. 13] to conclude $f=0$. Let $C(x, r)$ be the circle centered at $x$ and of radius $r$. Lemma 2.4 implies that all integrals of $f$ over all circles $C(x, r)$ for $x \in D(\varepsilon)$ and all $r>0$ are zero. In Helgason's argument on p. 13 and 14 of [31] he takes a derivative in the center, $x$, of $\int_{C(x, r)} f d s$ and uses the divergence theorem to show (by an inductive procedure) that $\int_{C(x, r)} f P d s=0$ for any polynomial $P$. The Stone Weierstrass Theorem is then used to show $f=0$ on $C(x, r)$. Since $r$ is arbitrary, $f=0$.

Our next corollary is a stronger result that follows from our proof.

Corollary 2.5. Let $\Omega^{\prime}$ be an open set and let $f$ be an $L^{1}$ function of compact support. Then, $f$ is determined everywhere by its values on $\Omega^{\prime}$ plus values of $R f$ for lines intersecting $\Omega^{\prime}$.

If $\Omega$ is an ROI and $\Omega^{\prime}$ is an open subset, then this corollary implies that knowing a function's line integrals on lines through $\Omega$ and its values on the smaller set $\Omega^{\prime}$ determine the function everywhere.

Proof of Corollary 2.5. One applies Theorem 2.2 to $\Omega^{\prime}$ and uses linearity: If $f_{1}$ and $f_{2}$ agree on $\Omega^{\prime}$ and have the same integrals on all lines intersecting $\Omega^{\prime}$, then $f=f_{1}-f_{2}$ is 0 on $\Omega^{\prime}$ (and so constant on $\Omega^{\prime}$ ) and $R f=0$ on lines meeting $\Omega^{\prime}$. Therefore, $f$ satisfies Hyp. 2.3. Then, Theorem 2.2 can be applied to conclude $f=0$ so $f_{1}=f_{2}$ everywhere.

Remark 2.6. Two important articles provide results that are closely related to Theorem 2.2. In [67], the authors prove that if a compactly supported square integrable function is constant on a circular ROI and $R f=0$ for lines meeting the ROI, then $f=0$ on the ROI.

In [64], the authors show that their TV minimization is valid for functions that are piecewise polynomial in the ROI, and they use Hilbert transform arguments to prove a variant of Theorem 2.2. One assumption in their theorems is less general than in Theorem 2.2; they assume $f$ is piecewise smooth [64, Conditions 1 and 4], and we assume $f \in L_{c}^{1}\left(\mathbb{R}^{2}\right)$. However, their second assumption is more general: they assume only that $f$ is piecewise polynomial in the ROI whereas, in Theorem 2.2, we assume $f$ is PC in the ROI.

A strengthening of the proof of Theorem 2.2 can be used to prove their theorem for $f \in L_{c}^{1}\left(\mathbb{R}^{2}\right)$, and it would generalize Theorem 2.2 to functions that are piecewise polynomial in the ROI. The changes to our proof are as follows: If $f$ is a polynomial in the small open set, $D(\varepsilon)$, then $f_{0}$ is an even polynomial in $r$; so if one takes derivatives $\frac{d}{d\left(r^{2}\right)}$ in $(2.3)$, the left-hand side will eventually become identically zero (and the right hand side starts at more negative power of $\left(s^{2}-r^{2}\right)$ ). Then (2.5) is true for all sufficiently large $n$ and the proof proceeds as in our proof of Theorem 2.2. 
A general uniqueness theorem such as Corollary 2.5 is not proven in that article.

Remark 2.7. These uniqueness results are surprising since there is a large null space for ROI tomography (e.g., Example 2.1). Furthermore, PC functions can be very close to null functions in $L^{1}$ norm [44]. This reinforces the point that our uniqueness result does not guarantee a stable inversion method, in general. In fact any inversion method could have instabilities, especially outside the region of interest. This is also apparent from microlocal analysis [49]; all singularities $f$ in the ROI are visible from ROI data, but some singularities of $f$ at points outside the ROI will not be stably visible (those not normal to lines in the ROI data set).

So, any reconstruction method that uses PC functions must really "use" them in their assumptions and in the reconstruction method. The Mumford Shah methods in [37,39] and the total variation methods in [66] do use them. We use this assumption in our algorithm by assuming our function can be written as a finite sums of Haar basis functions (see (3.1)).

\section{The Setup}

Since we consider region of interest CT, we want to reconstruct a function $f$ from local projection data. We assume that the (convex hull of the) support of $f$ is contained in $D \subset \mathbb{R}^{2}$. Please note that it might be the case that the function $f$ represents an object that has inclusions with value zero (e.g., holes in a workpiece in non-destructive testing) and hence, we consider the convex hull of the support. We assume that $D$ is a square that can be easily partitioned by Haar wavelets. For simplicity, we further assume that the region of interest, denoted by $\Omega$, is a circle contained in $D$. Thus, we assume that the same number of projections is used for every direction. The circle is not required to be in the center of the domain $D$ and can vary in size. As defined in the introduction, see (1.3), we consider a restricted Radon operator, taking projections only along lines intersecting the ROI,

$$
R_{\lim } f(\omega, s):= \begin{cases}R f(\omega, s) & \text { if } \ell(\omega, s) \cap \Omega \neq \emptyset \\ 0 & \text { else. }\end{cases}
$$

Theorem 2.2 shows that piecewise constant functions can be recovered uniquely from the measurements $z_{\text {lim }}$ taken from all lines $\mathcal{L}$ intersecting the region of interest (at least

for continuous data). It therefore seems reasonable to restrict our attention to this type of functions, that is, functions which can be represented as a finite linear combination of characteristic functions (see (1.1)). As we want to use a reconstruction approach based on orthonormal basis functions, we will restrict the space of admissible functions even further: Consider the orthonormal Haar-Wavelet basis

$$
\mathcal{H}=\left\{\phi_{j k} \mid j \in \mathbb{N}, k \in \mathbb{N}\right\} .
$$

Wavelet bases were originally defined as basis functions for $L_{2}(\mathbb{R})$, see the standard references $[10,13,36,43,61]$ on wavelets. Using tensor wavelets, orthonormal bases for higher dimensions can easily be obtained. The construction of orthonormal wavelets on a bounded domain usually requires a change in the boundary functions. In contrast to the decomposition on unbounded regions, for bounded domains one has on each level $j$ only a finite number $K(j)$ of shifts, see, e.g., [13]. 
Thus we will consider the space

$$
\mathrm{PCH}_{N}=\left\{f \in L_{2}(D): f(x)=\sum_{j=0}^{N} \sum_{k=0}^{K(j)}\left\langle f, \phi_{j k}\right\rangle \phi_{j k}(x)\right\} .
$$

Note that $\mathrm{PCH}_{N}$ contains

$$
L=\sum_{j=0}^{N} K(j)
$$

basis functions. We will call such functions $\mathrm{PCH}$ functions (or $\mathrm{PCH}_{N}$ functions when we have a specific $N$ in mind).

Please note that the admissible functions $f \in \mathrm{PCH}_{N}$ have a finite decomposition w.r.t. the Haar basis, i.e., they are sparse w.r.t. to the Haar basis decomposition. As each Haar basis function is itself piecewise constant, a function $f \in \mathrm{PCH}_{N}$ is a piecewise constant function. On the other hand, not every piecewise constant function can be represented by functions in $\mathrm{PCH}_{N}$, which means we restrict ourselves to a subspace only. However, we expect a good reconstruction for all piecewise constant functions that can be well approximated by functions from $\mathrm{PCH}_{N}$. So far, we have considered the operator $R_{\lim }: L_{2}(D) \rightarrow L_{2}\left(\mathbb{R} \times S^{1}\right)$. Defining

$$
\begin{gathered}
F: \mathrm{PCH}_{N} \subset L_{2}(D) \rightarrow \ell_{2}\left(\mathbb{R}^{L}\right) \\
F f:=\left(\left\langle f, \phi_{j k}\right\rangle\right)_{j=1, \ldots, N, k=0, \ldots K(j)},
\end{gathered}
$$

we obtain for $c \in \ell_{2}\left(\mathbb{R}^{L}\right)$

$$
F^{*} c=\sum_{j, k} c_{j k} \phi_{j k}
$$

and finally define

$$
\begin{aligned}
& R_{l}: \ell_{2} \rightarrow \mathcal{R}\left(R_{l}\right) \subset L_{2}\left(\mathbb{R} \times S^{1}\right) \\
& R_{l}:=R_{\lim } F^{*}
\end{aligned}
$$

\section{TikHONOV REgUlaRizATION WITH SPARSITY CONSTRAintS}

Regularization methods obtain an approximation to the solution of an ill-posed problem by replacing the unstable inversion. For an ill-posed problem with forward mapping $A: X \rightarrow Y$ a regularization is a mapping $T_{\alpha}: Y \rightarrow X$ and a parameter choice rule for $\alpha \in \mathbb{R}_{+}$such that the regularized solutions converge to the solution of the original problem.

Standard Tikhonov regularization [20] obtains an approximation to the solution by solving the surrogate problem

$$
f_{\alpha}^{\delta}=\underset{f}{\operatorname{argmin}}\left\{\left\|z_{\lim }^{\delta}-R_{\lim } f\right\|_{L_{2}\left(S^{1} \times \mathbb{R}\right)}^{2}+\alpha\|f\|_{L_{2}}^{2}\right\} .
$$

Alternatively, $f_{\alpha}^{\delta}$ is obtained as solution of the equation

$$
\left(R_{\lim }^{*} R_{\lim }+\alpha I\right) f=R_{\lim }^{*} z_{\lim }^{\delta} .
$$

As we assume that our solution has a sparse representation with respect to the Haar basis, we will use Tikhonov regularization with a sparsity promoting penalty, see especially $[14,27$, $41,50,53]$. 
This could be a (power of a) Besov norm or more general a (power of a) weighted $\ell_{p}$-norm of the wavelet coefficients. Denoting by $c_{j k}$ the wavelet coefficients of the function $f$, that is, $c_{j k}=F f=\left\langle f, \phi_{j k}\right\rangle$, we obtain an approximation of the solution as minimizer of the functional

$$
f=\underset{f \in \mathrm{PCH}}{\operatorname{argmin}}\left\{\left\|R_{\lim } f-z_{\lim }^{\delta}\right\|_{L_{2}\left(S^{1} \times \mathbb{R}\right)}^{2}+\alpha\|F f\|_{p, \omega}^{p}\right\}
$$

where

$$
\|F f\|_{p, \omega}^{p}=\sum_{j=0}^{N} \sum_{k=0}^{K(j)} \omega_{j k}\left|c_{j k}\right|^{p}
$$

is a weighted $\ell_{p}$-norm with strictly positive weights $0<C \leq \omega_{j k}$. Please note that for $p>1$ the functional is strictly convex and has a unique minimizer. For $p=1$ uniqueness is given, e.g., if the operator is injective. Since the penalty term works on sequences we rewrite the functional with the help of the operator $R_{l}$ as

$$
c=\underset{c \in \ell_{2}}{\operatorname{argmin}}\left\{\left\|R_{l} c-z_{\lim }^{\delta}\right\|^{2}+\alpha \sum_{j=0}^{N} \sum_{k=0}^{K(j)} \omega_{j k}\left|c_{j k}\right|^{p}\right\} .
$$

The minimizer of the above functionals will depend on the following parameters: the regularization parameter $\alpha$, the weights $\omega:=\left(\omega_{j k}\right)_{j k}$, the norm parameter $p$ and the possibly noisy data $z_{\lim }^{\delta}$. Taking all parameters into account we denote the minimizer of (4.2) by $c_{\alpha, \omega, p, \delta}^{\star}$. The corresponding function will be denoted by $f_{\alpha, \omega, p, \delta}^{\star}$ and we have $f_{\alpha, \omega, p, \delta}^{\star}=F^{*} c_{\alpha, \omega, p, \delta}^{\star}$ and $f_{\alpha, \omega, p, \delta}^{\star}$ is the minimizer of (4.1). For the minimization of the functional, several methods do exists, e.g., the method of surrogate functionals [14,50]. In that method, the functional is replaced by a sequence of easier-to-minimize "surrogate" functionals such that the successive minimizers converge to the desired limit $f_{\alpha, \omega, p, \delta}^{\star}$.

Regularization theory is concerned with the convergence of the regularized solutions (in our case, the minimizer of the Tikhonov functional with a sparsity constraint) to the solution of the equation. We have the following result:

Theorem 4.1. Assume that $A: X \rightarrow Y$ is a bounded operator between Hilbert spaces $X, Y$, $1 \leq p \leq 2$, and that $0<c<\min _{\lambda} w_{\lambda},\left\{\omega_{\lambda}\right\}_{\lambda \in \Lambda}=\omega$. For $p=1$ assume further $\mathcal{N}(A)=\{0\}$. Let $f_{\alpha, \omega, p, \delta}^{\star}$ be the unique minimizer of (4.1) for given data $z^{\delta}$ with $\left\|z-z^{\delta}\right\| \leq \delta$ and $\alpha>0$. If $\alpha=\alpha(\delta)$ satisfies the requirements

$$
\lim _{\delta \rightarrow 0} \alpha(\delta) \rightarrow 0 \quad \text { and } \quad \lim _{\delta \rightarrow 0} \frac{\delta^{2}}{\alpha(\delta)}=0,
$$

then we have,

$$
\lim _{\delta \rightarrow 0}\left[\sup _{\left\|z-z^{\delta}\right\| \leq \delta}\left\|f_{\alpha, \omega, p, \delta}^{\star}-f^{\dagger}\right\|\right]=0,
$$

i.e., the regularized solutions converge to the solution of the equation $A f^{\dagger}=z$ that has minimal value of $\|\cdot\|_{p, \omega}^{p}$. 
This theorem was first proven in the seminal paper [14]. Please note that the condition on the injectivity of $A$ for $p=1$, which is in turn needed to prove the existence of a unique minimizer of the Tikhonov functional, has been slightly weakened in [7]. We may add that results for different parameter choice rules do exist, e.g., for the discrepancy principle [1]. We also would like to mention convergence speed results, which require, e.g., source conditions for the solution $[2,3,8,14,26,27,41,51]$. Convergence and convergence rates for sparse regularization in a Bayes setup have been recently investigated in [25].

We immediately have:

Corollary 4.2. Tikhonov regularization with sparsity penalty for region of interest CT with continuous data over the space $\mathrm{PCH}_{N}$ is a regularization method.

Proof. According to Theorem 2.2 we have that the region of interest CT operator is injective. Hence, the minimizer of (4.1) is unique. $\mathrm{As}^{\mathrm{PCH}_{N}}$ and the data space are Hilbert spaces, the corollary follows from Theorem 4.1.

\section{Parameter and Weight Choices}

We aim for an approximate solution to the problem of solving $R_{\lim } f=z_{\lim }$ from noisy data $z_{\lim }^{\delta}$ when $f$ is a piecewise constant function. We gain such an approximate solution in the space $\mathrm{PCH}$ of piecewise constant functions spanned by the Haar-wavelet by computing the wavelet coefficients as given in (4.2), i.e.,

$$
\begin{aligned}
f & =\sum_{j k} c_{j k} \phi_{j k} \text { with } \\
c_{j k} & =\underset{c \in \ell_{2}}{\operatorname{argmin}}\left\{\left\|R_{l} c-z_{\lim }^{\delta}\right\|_{L_{2}\left(S^{1} \times \mathbb{R}\right)}^{2}+\alpha \sum_{j=0}^{N} \sum_{k=0}^{K(j)} \omega_{j k}\left|c_{j k}\right|^{p}\right\} .
\end{aligned}
$$

In the following we discuss the choice of the regularization parameter $\alpha$, the weights $\omega_{j k}$ and the $\ell_{p}$-norm. It is well-known that an $\ell_{p}$-norm with $p<2$ promotes sparsity, whereas $p \geq 1$ ensures convexity. The theoretical results mentioned at the end of the previous section hold true for all $1 \leq p \leq 2$ and for all sequences of weights strictly bounded away from zero. For our numerical experiments in Section 6 we set $p=1$. For the choice of weights we consider three types of basis functions in the space PCH. The first type are basis functions with support either containing the region of interest (approximation, coarse wavelets), or being contained in the region of interest (detail, fine wavelet). These basis functions assemble the reconstruction in the ROI and hence, the coefficients should be computed as accurately as possible. We choose the weight to be equal to 1 for these basis functions. The second type are basis functions whose support does not overlap the region of interest (details, fine wavelets and coarser wavelets away from the ROI). Although these functions do not affect the actual reconstruction in the ROI they have an influence on the data fit term (the first term) of (4.2) and hence on the values of the coefficients for the basis functions of the first type. We choose a fixed value for the corresponding weights. In the numerical examples we experiment with different values for this outside weight, e.g., 2, 5 and 10, but also 0.5. With a weight larger than 1 we make sure that the coefficients outside the ROI are constrained but can still contribute to the reconstruction. In doing so we make sure that small, but significant details are still allowed to contribute to the reconstruction. The larger the weight for these 'outside' 
basis functions the sparser the reconstruction, see the examples and discussion in the next section. The last type of basis functions are those that overlap the region of interest without belonging to the first type. For these functions we define intermediate weights depending on the size of the intersection (the size of the shared support). The weight interpolates between 1, the inside weight, and the value chosen for the outside weight (empty intersection).

Splitting the set of indices $\Gamma$ for the basis functions according to the position of the basis functions with respect to the ROI in three sets, namely $\Gamma_{\text {in }}, \Gamma_{\text {out }}$ and $\Gamma_{\text {rim }}$, the regularization functional can be written as

$$
c_{j k}=\underset{c \in \ell_{2}}{\operatorname{argmin}}\left\{\left\|R_{l} c-z_{\lim }^{\delta}\right\|_{L_{2}}^{2}+\alpha\left(\sum_{j k \in \Gamma_{\text {in }}}\left|c_{j k}\right|^{p}+\sum_{j k \in \Gamma_{\text {rim }}} \omega_{j k}\left|c_{j k}\right|^{p}+\omega_{\text {out }} \sum_{j k \in \Gamma_{\text {out }}}\left|c_{j k}\right|^{p}\right)\right\} .
$$

Other choices for the interpolation between the weight inside the ROI and the outside are, of course, possible. Furthermore, one can also use level dependent weights, thus using a more distinct penalization of coarse and fine details.

For the minimization of (5.1) we use the method of surrogate functionals $[14,50]$ : The functional is replaced by a sequence of easier-to-minimize "surrogate" functionals such that the successive minimizers converge to the desired limit. In each step of this iterative procedure soft shrinkage is applied.

\section{Numerical Results}

We consider three examples: (i) a purely academic, and very sparse wavelet example, (ii) the MCAT torso phantom [59,63], and (iii) the Shepp-Logan head phantom. The wavelet example is constructed to demonstrate the ability of the proposed method to numerically reconstruct everything (even outside the ROI). Even though theoretically, see Theorem 2.2, the reconstruction of $\mathrm{PC}$ functions is possible from ROI data, in numerical computation the data do not fulfill the requirements of the theorem. The data are sampled, i.e., only finitely many projections along finitely many lines (through the region of interest) are taken. The case that projections are taken from only very few directions $[29,34,48]$ is not considered in the present work.

The implementation is done in Matlab. All data are simulated data. For the reconstruction method we choose the Haar (db1) wavelet and $p=1$. Different sets of weights and regularization parameter are chosen. The approximation quality of the computed reconstruction $f_{\text {reco }}$ is measured by computing the relative $L_{2}$-error with respect to the true target image $f_{\text {true }}$, both for the whole image $D$ as well as for the ROI $\Omega$ :

$$
\delta_{\Omega}:=\frac{\left\|f_{\text {true }}-f_{\text {reco }}\right\|_{L_{2}(*)}}{\left\|f_{\text {true }}\right\|_{L_{2}(*)}}
$$

The weights are computed numerically as follows: given the finest possible wavelet decomposition (Matlab command wmaxlev), we reconstruct from all possible delta-sequences and check the overlap of this reconstructions with the ROI.

The regularization parameter is chosen manually to get small reconstruction errors and visually best looking results. Stopping criteria such as Morozov's discrepancy principle or the S-curve method of [29] are still to be examined. 
6.1. A very sparse wavelet example. We consider an academic example built from 8 wavelets of different size, see Figure 1, and the first row of Table 1 for the values of the coefficients. An important feature is the small wavelet - corresponding to a fine detail with high intensity - outside the region of interest. Our proposed method allows the reconstruction of fine details outside the ROI; and Figure 1 as well as Tables 1 and 2 demonstrate this. Table 1 shows the values of the wavelet coefficients for the original function and for the solutions to the weighted wavelet functional with different values of the regularization parameter $\alpha$. The number of nonzero coefficients and their values for decreasing $\alpha$ indicate convergence of the coefficients to the true solution. Table 2 shows reconstructions errors in the ROI and in the whole image domain. The proposed method is able to achieve a very small relative reconstruction error both in the region of interest and in the whole image domain.

In [48] a wavelet based method is proposed that deletes fine detail levels outside the region of interest in order to get a regularized and sparse solution in the ROI. With this method, the small detail cannot be reconstructed as soon as more than 1 detail level outside the ROI are deleted, see the second row of Figure 1. From the reconstruction errors given in Table 3 one can see that also with this approach a very small reconstruction error in the ROI can be achieved. This happens, however, at the cost of the sparseness of the reconstruction (see, e.g., the first row of Table 3). The second part of Table 3 with two detail levels deleted outside the ROI shows that increasing the regularization parameter indeed enforces sparsity (decrease in the number of nonzero wavelet coefficients) but at a cost to the data fit. It also shows that without the small detail the error of the reconstruction in the whole image domain is quite large. Although one might be only interested in the reconstruction in the ROI, with regard to Theorem 2.2, it seems a good idea to also reconstruct the outside as good as possible.

The influence of the regularization parameter can also be observed for the proposed weighted wavelet method. If the regularization parameter is chosen too small the reconstruction becomes less sparse (Table 2 , the pair $\left(\omega_{\text {out }}, \alpha\right)=\left(10,1 \cdot 10^{-7}\right)$ ), whereas a too large regularization parameter subdues small details (Table 2 , the pair $\left(\omega_{\text {out }}, \alpha\right)=\left(10,5 \cdot 10^{-5}\right)$ ).

Please note that choosing a different outside weight is not just a simple scaling that can be balanced by the regularization parameter but effects the quality of the reconstructed solution. This is due to the fact that the minimizer of (5.1) is computed using a nonlinear shrinkage method that is affected by a change in the weights. As an example, we point out the pairs $\left(\omega_{\text {out }}, \alpha\right)=\left(10,5 \cdot 10^{-7}\right)$ and $\left(\omega_{\text {out }}, \alpha\right)=\left(5,1 \cdot 10^{-6}\right)$ in Table 2 with the different reconstruction errors $\delta_{\mathrm{ROI}}\left(10,5 \cdot 10^{-7}\right)=0.379 \%$ and $\delta_{\mathrm{ROI}}\left(5,1 \cdot 10^{-6}\right)=0.417 \%$ respectively. Further evidence of the existence of an 'optimal pair' of weight and parameter can be found also in Table 5.

6.2. Reconstructions from the MCAT torso phantom. The proposed algorithm is applied to numerically generated ROI-CT data for the standard mathematical cardiac torso (MCAT) phantom [59,63], see Figure 2. The piecewise constant density function $\mu$ represents a section through a simplified model of a human torso: Figure 2 (left) shows spine (no.4), spinal canal (no.5), the lungs (no.3 and no.6), the surrounding tissue (no.2) and the exterior (no.1). The table in Figure 2 gives the exact density values $\mu_{i}$ of the piecewise constant torso phantom. The tomography data are generated by a Matlab implementation of the limited Radon operator (1.2) where 160 offsets and 159 directions are used. The region of 

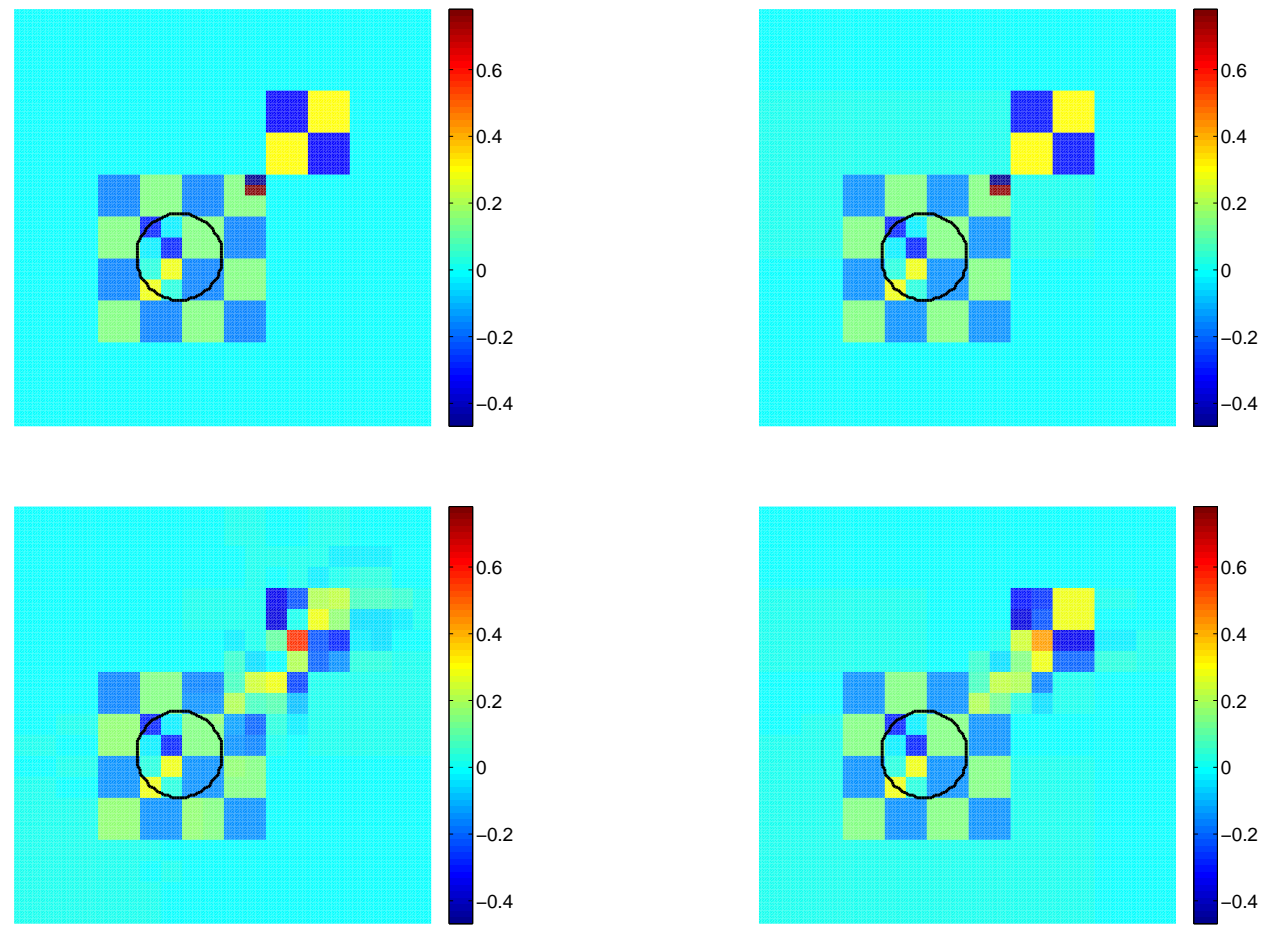

Figure 1 - A very sparse wavelet example with region of interest. First row left: Original. Right: reconstruction from region of interest data with the proposed weighted wavelet method and regularization parameter $\alpha=5 \cdot 10^{-7}$ and outer weight equal to 10 . Second row: Reconstructions with the linear shrinkage reconstruction as in [48]; outside of the ROI the 2 finest detail levels are missing. Left: $\alpha=1 \cdot 10^{-6}$. Right: $\alpha=5 \cdot 10^{-6}$. See also Table 3 . The colors are normalized for better comparison.

interest is an off-center circle including part of the spine and the spinal canal as well as some part of the left lung, see Figure 2 (right).

In this example we choose a region of interest that includes the bigger part but not all of the spine where the spine has the highest attenuation coefficient. We want to test our method in the situation that right outside the region of interest a small area with high attenuation exists.

Figure 3 shows some example reconstructions with the db1-wavelet, the regularization parameter $\alpha=5 \cdot 10^{-8}$ and different weights. Reconstruction errors for different regularization parameter and weights are listed in Table 5. Please note that the listed combinations of weights and regularization parameter indicate that there is a 'best pair', meaning a combination of $\alpha$ and $\omega_{\text {outside }}$ that gives the smallest relative reconstruction error within the ROI. This will be the topic of future work. E.g., we plan to adapt the "S-curve method" [40,48], 


\begin{tabular}{|c|c|c||c|}
\hline$\alpha$ & $\omega_{\text {out }}$ & nnz & $100 \cdot\left(c_{1}, c_{2}, \ldots, c_{8}\right)$ \\
\hline \hline \multicolumn{2}{|c|}{ original } & 8 & $3.125,3.125,3.125,3.125,6.250,1.250,1.250,3.125$ \\
\hline $5 \cdot 10^{-7}$ & & 8 & $3.112,3.113,3.111,3.117,6.215,1.244,1.246,3.028$ \\
$1 \cdot 10^{-6}$ & \multirow{3}{*}{10} & 8 & $3.099,3.102,3.097,3.110,6.181,1.238,1.242,2.931$ \\
$5 \cdot 10^{-6}$ & & 8 & $2.999,3.008,2.983,3.049,5.904,1.190,1.209,2.155$ \\
$1 \cdot 10^{-5}$ & & 8 & $2.874,2.891,2.841,2.973,5.557,1.130,1.170,1.184$ \\
\hline $1 \cdot 10^{-6}$ & & 8 & $3.112,3.113,3.111,3.117,6.215,1.243,1.244,3.028$ \\
$5 \cdot 10^{-6}$ & \multirow{3}{*}{5} & 8 & $3.061,3.064,3.053,3.086,6.077,1.213,1.222,2.640$ \\
$1 \cdot 10^{-5}$ & & 8 & $2.996,3.004,2.981,3.047,5.905,1.176,1.194,2.156$ \\
\hline
\end{tabular}

Table $1-$ A very sparse wavelet example. Reconstructions with the proposed weighted wavelet method. Values of the wavelet coefficients of the true phantom and the reconstruction for different values of the regularization parameter and $\omega_{\text {out }}=5$ and 10 .

\begin{tabular}{|c|c||c|c|c|}
\hline$\alpha$ & $\omega_{\text {out }}$ & nnz & $\delta_{\mathrm{ROI}}$ in $\%$ & $\delta_{\mathrm{D}}$ in $\%$ \\
\hline \hline 0 & no influence & 22396 & 10.731 & 72.708 \\
\hline \hline $1 \cdot 10^{-7}$ & & 181 & 0.216 & 34.525 \\
$5 \cdot 10^{-7}$ & & 8 & 0.379 & 1.114 \\
$1 \cdot 10^{-6}$ & & 8 & 0.758 & 2.221 \\
$5 \cdot 10^{-6}$ & \multirow{2}{*}{} & 8 & 3.789 & 11.105 \\
$1 \cdot 10^{-5}$ & & 8 & 7.578 & 22.211 \\
$5 \cdot 10^{-5}$ & & 7 & 38.404 & 52.846 \\
\hline \hline $1 \cdot 10^{-7}$ & & 571 & 1.410 & 45.600 \\
$5 \cdot 10^{-7}$ & & 45 & 0.235 & 12.603 \\
$1 \cdot 10^{-6}$ & 5 & 8 & 0.417 & 1.115 \\
$5 \cdot 10^{-6}$ & & 8 & 2.084 & 5.560 \\
$1 \cdot 10^{-5}$ & & 8 & 4.167 & 11.120 \\
\hline
\end{tabular}

Table 2 - A very sparse wavelet example. Reconstructions with the proposed weighted wavelet method. Relative $\mathrm{L}_{2}$-error in the ROI and in the image domain for different values of the regularization parameter and $\omega_{\text {out }}=5$ and 10 .

a sparsity-based choice rule for the parameter $\alpha$ based on a priori knowledge of the number of nonzero wavelet coefficients in $\mu$, e.g., from an anatomical atlas.

Figure 4 and Table 6 allow a comparison of our weighted wavelet method with the linear shrinkage outside of the ROI as done in [48]. One can see that with the linear shrinkage method the smallest reconstruction error is achieved when only the finest detail level is deleted outside the region of interest. The relative reconstruction error in the ROI is then $14.93 \%$ where as the weighted wavelet method achieves a reconstruction error of $10.34 \%$ with a comparable amount of nonzero wavelet coefficients. The first row of Figure 4 shows the influence of the missing detail levels outside the ROI. The more detail levels are missing, the 


\begin{tabular}{|c|c||c|c|c|}
\hline$\alpha$ & no. of deleted levels & nnz & $\delta_{\mathrm{ROI}}$ in $\%$ & $\delta_{\mathrm{D}}$ in $\%$ \\
\hline \hline $5 \cdot 10^{-7}$ & & 247 & 0.195 & 42.3 \\
$1 \cdot 10^{-6}$ & 1 & 133 & 0.226 & 32.41 \\
$5 \cdot 10^{-6}$ & & 8 & 0.864 & 1.153 \\
$1 \cdot 10^{-5}$ & & 8 & 1.727 & 2.302 \\
\hline \hline $5 \cdot 10^{-7}$ & & 162 & 0.972 & 57.507 \\
$1 \cdot 10^{-6}$ & & 109 & 0.683 & 53.552 \\
$5 \cdot 10^{-6}$ & 2 & 33 & 1.030 & 39.433 \\
$1 \cdot 10^{-5}$ & & 23 & 2.267 & 36.042 \\
$5 \cdot 10^{-5}$ & & 11 & 8.872 & 33.756 \\
\hline
\end{tabular}

Table 3 - A very sparse wavelet example. Reconstructions with the linear shrinkage method of [48]. Relative $\mathrm{L}_{2}$-error in the ROI and in the image domain for different values of the regularization parameter and different numbers of deleted detail levels outside the ROI.

\begin{tabular}{|c|c||c|c|c|}
\hline$\alpha$ & $\omega_{\text {out }}$ & nnz & $\delta_{\text {ROI }}$ in $\%$ & $\delta_{\mathrm{D}}$ in $\%$ \\
\hline \hline $1 \cdot 10^{-6}$ & & 268 & 11.987 & 4.206 \\
$5 \cdot 10^{-6}$ & & 15 & 2.094 & 5.612 \\
$7 \cdot 10^{-6}$ & 5 & 9 & 2.615 & 8.120 \\
$1 \cdot 10^{-5}$ & & 8 & 0.406 & 10.952 \\
$5 \cdot 10^{-5}$ & & 7 & 20.705 & 39.235 \\
\hline
\end{tabular}

Table 4 - A very sparse wavelet example. Reconstructions with the proposed weighted wavelet method from data with $10 \%$ relative noise. Relative $\mathrm{L}_{2}$-error in the $\mathrm{ROI}$ and in the image domain for different values of the regularization parameter and $\omega_{\text {out }}=5$.

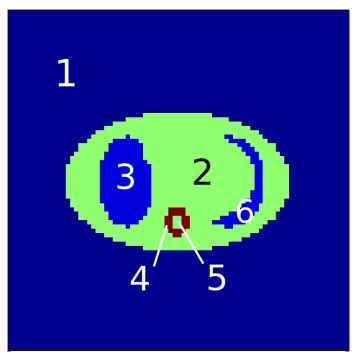

\begin{tabular}{|c|c|}
\hline $\mathrm{j}$ & $\mu_{j}$ \\
\hline 1 & 0 \\
2 & 0.0600 \\
3 & 0.0100 \\
4 & 0.1200 \\
5 & 0.0600 \\
6 & 0.0100 \\
\hline
\end{tabular}

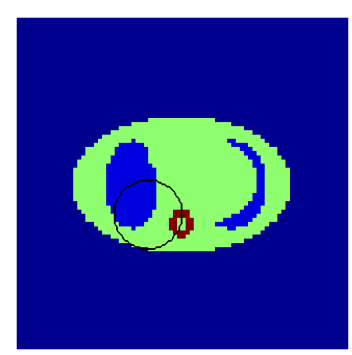

Figure 2 - Density function from a torso phantom with numbered domains (left). Density values of the domains (middle). Torso phantom with region of interest (right).

larger the 'pixels' (squares representing the basis functions that are used) get outside the ROI. However, the difference between the linear shrinkage method [48] and the proposed weighted wavelet method is that for the weighted wavelet method, small wavelets are always allowed outside the ROI, and they show up in the spine region. From the zoom into the difference 
of reconstruction and true solution (Figures 3 and 4, lower row), one can see that the part of the spine that is outside the ROI is much better reconstructed for the weighted wavelet method. As this is an area of high attenuation, the observations about the reconstruction error indicate that, indeed, its reconstruction influences the overall reconstruction.
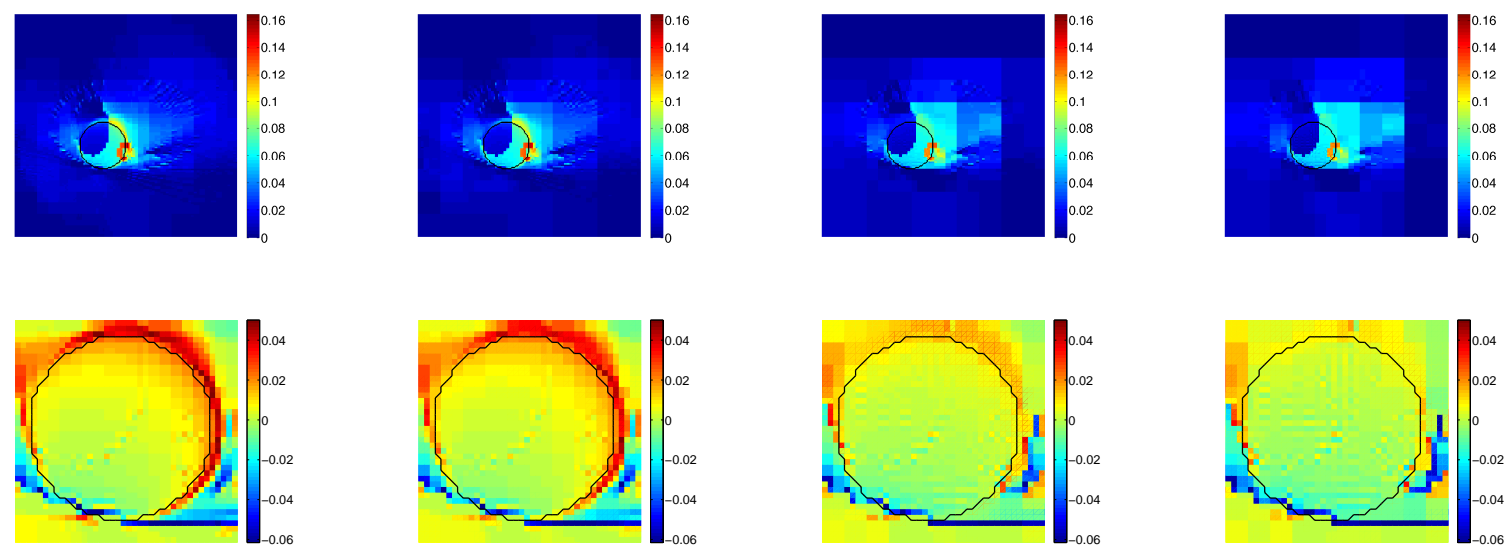

Figure 3 - Reconstructions from noise-free data with the proposed weighted wavelet method; $\alpha=5 \cdot 10^{-8}$. Upper row: reconstructions. Lower row: difference of reconstruction and true image with zoom into the ROI. From left to right: Weight outside 0.5, 1 (i.e., all weights are equal to 1), 5 and 10 . The colors are normalized so that all images are shown within the same range.

\begin{tabular}{c|l|cccccccccc}
\hline$\alpha$ & $\omega_{\text {out }}$ & 0.25 & 0.5 & 1 & 2 & 5 & 10 & 15 & 20 & 25 & 30 \\
\hline \hline \multirow{5}{*}{$5 \cdot 10^{-7}$} & nnz & 1064 & 719 & 512 & 355 & 239 & 185 & - & - & & \\
& $\delta_{\text {ROI }}$ & 18.10 & 17.09 & 17.56 & 18.98 & 21.25 & 23.77 & - & - & & \\
& $\delta$ & 58.48 & 57.05 & 56.62 & 59.05 & 60.66 & 61.69 & - & - & & \\
\hline \multirow{5}{*}{$5 \cdot 10^{-8}$} & nnz & - & 3049 & 1957 & 1358 & 916 & 778 & 690 & - & - & \\
& $\delta_{\text {ROI }}$ & - & 17.21 & 15.79 & 13.38 & 10.34 & 11.63 & 13.73 & - & - & \\
& $\delta$ & - & 59.82 & 58.67 & 57.21 & 55.80 & 56.38 & 57.97 & - & - & \\
\hline \multirow{5}{*}{$1 \cdot 10^{-8}$} & nnz & - & 8536 & 5775 & 3763 & 2322 & 1659 & 1457 & 1344 & 1247 & 1194 \\
& $\delta_{\text {ROI }}$ & - & 19.23 & 18.80 & 17.98 & 16.23 & 14.12 & 12.81 & 12.30 & 12.32 & 12.74 \\
& $\delta$ & - & 61.43 & 60.90 & 60.14 & 58.67 & 57.21 & 56.39 & 56.05 & 55.86 & 55.95 \\
\hline
\end{tabular}

Table 5 - Reconstructions from noise-free data with the proposed weighted wavelet method for different values of the regularization parameter and the outside weight; $\omega_{\text {out }}=1$ refers to the case where all weights are equal to 1, i.e., a standard Besov penalty.

Comparing the reconstructions of the proposed weighted wavelet method for the very sparse wavelet example and the MCAT phantom, Figures 1 and 3, one could observe that for the very sparse wavelet example the whole function, i.e., also the exterior is reconstructed 

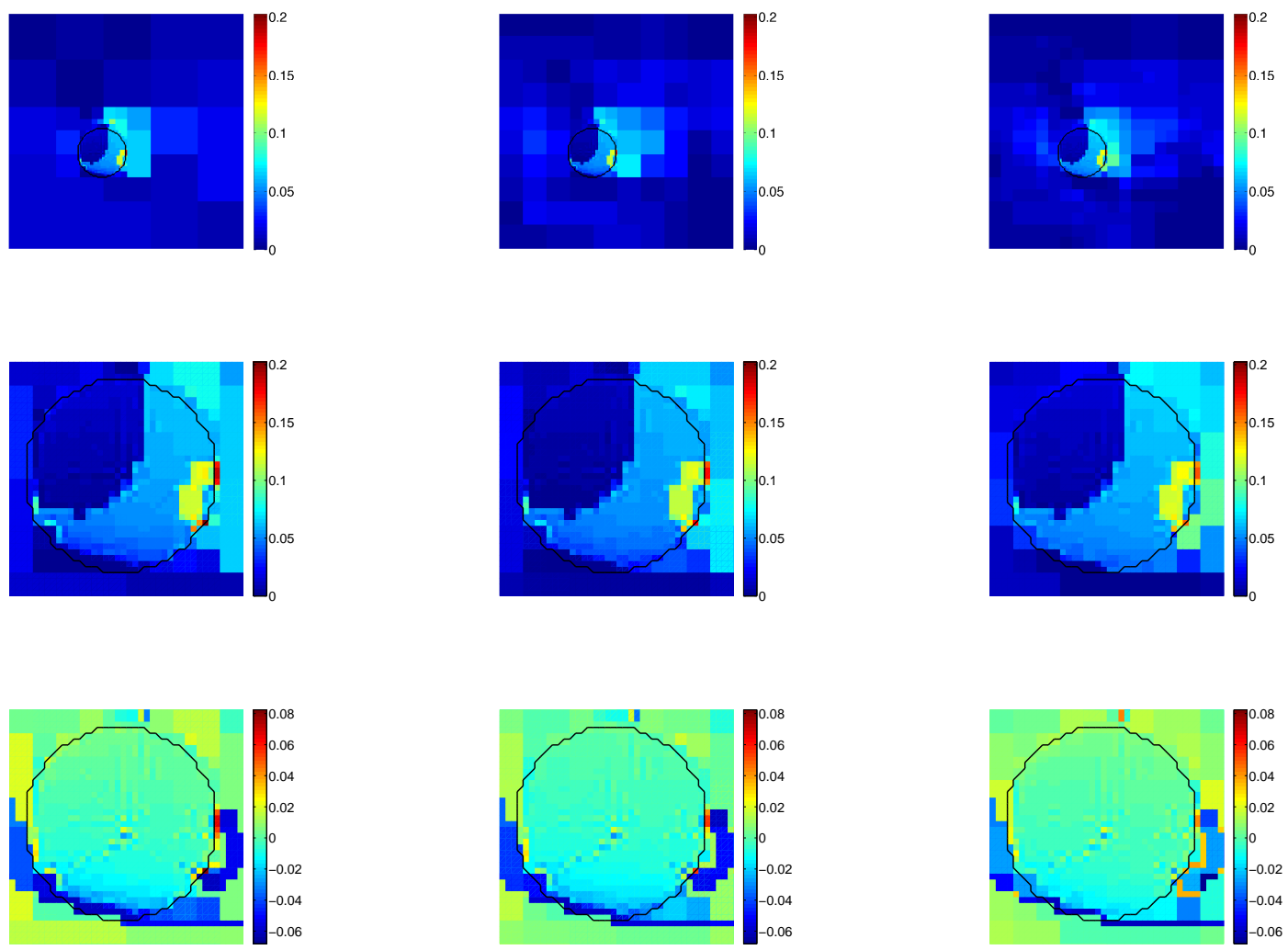

Figure 4 - Reconstructions from noise-free data with db1 and the linear shrinkage method outside the ROI as introduced in [48]. Upper row: reconstructions. Middle row: zoom into reconstructed ROI. Lower row: difference and zoom into ROI. Left: 4 from 7 detail levels are used outside the ROI. Middle: 5 from 7 detail levels are used outside the ROI. Right: 6 from 7 detail levels are used outside the ROI. The colors are normalized so that all images are shown within the same range.

extremely well, whereas this is not the case for the MCAT phantom. This is a result of using discrete, sampled data in contrast to continuous data in the theoretical result. For the very sparse example the amount of data is sufficient to also reconstruct the exterior whereas this is not the case for the MCAT phantom with its much higher structure. This also reflects the high instability for reconstructing outside the ROI.

6.3. Reconstructions for the Shepp-Logan head phantom. In order to compare our method to the one from [66] we apply it to the same 2-dimensional modified Shepp-Logan head phantom. The exact specifications are taken from [66, Table 1] and the description given in the text. Hence, we use a $256 \times 256$ matrix with a region of interest as shown in Figure 5, left. We reconstruct the ROI by the proposed weighted wavelet method and show the results in Figure 5, right. The same profile lines as in [66] are also shown to allow a comparison, see Figure 6 . We would like to point out that the region of interest chosen in [66] is rather large in comparison to the phantom. This explains that the weights have 


\begin{tabular}{c|c|ccc}
\hline$\alpha$ & \# deleted levels & 1 & 2 & 3 \\
\hline \hline \multirow{3}{*}{$5 \cdot 10^{-7}$} & $\mathrm{nnz}$ & 364 & 298 & 265 \\
& $\delta_{\mathrm{ROI}}$ & 20.30 & 23.06 & 28.81 \\
& $\delta$ & 60.75 & 63.16 & 69.38 \\
\hline \multirow{3}{*}{$1 \cdot 10^{-7}$} & $\mathrm{nnz}$ & 819 & 618 & 500 \\
& $\delta_{\mathrm{ROI}}$ & 14.93 & 18.00 & 28.15 \\
& $\delta$ & 61.86 & 64.56 & 71.47 \\
\hline \multirow{3}{*}{$5 \cdot 10^{-8}$} & $\mathrm{nnz}$ & 1155 & 839 & 680 \\
& $\delta_{\mathrm{ROI}}$ & 15.86 & 18.68 & 29.30 \\
& $\delta$ & 63.28 & 65.71 & 72.45 \\
\hline \multirow{3}{*}{$1 \cdot 10^{-8}$} & $\mathrm{nnz}$ & 1998 & 1200 & 964 \\
& $\delta_{\mathrm{ROI}}$ & 19.25 & 21.49 & 31.73 \\
& $\delta$ & 65.44 & 67.16 & 73.52 \\
\hline
\end{tabular}

Table 6 - Reconstructions from noise-free data with db1 and the linear shrinkage method outside the ROI as introduced in [48].

a fairly small effect in this computations. We have also point out that our reconstructions are comparable to those in [66] but with a much rougher angular sampling (255 equi-angular views compared to 1300 equi-angular views in [66]).
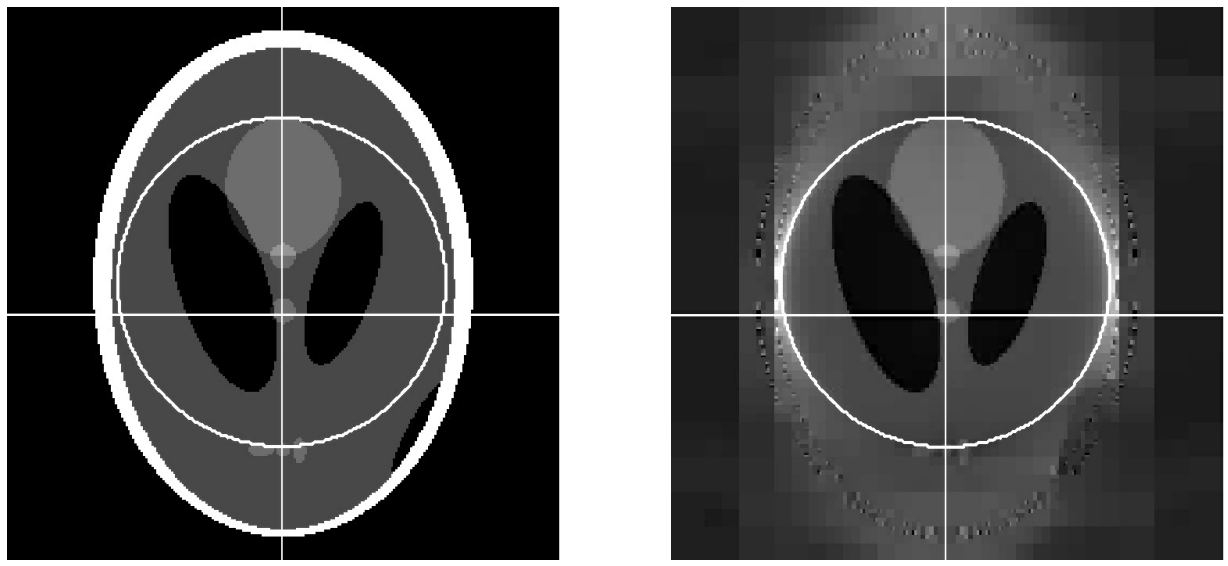

Figure 5 - Shepp-Logan phantom. Left: the original phantom as used in [66] with lines indicating the ROI and two profiles. Right: reconstruction using the proposed weighted wavelet method with outer weight equal to 2 and $\alpha=5 \cdot 10^{-7}$. The images were modified (contrast, light) to enhance visibility. The same grayscale is used for both pictures.

\section{Conclusions}

We presented a sparsity promoting reconstruction method for ROI tomography of piecewise constant functions using a Haar wavelet basis and a weighted $\ell_{p}$-penalty. Theorem 2.2 

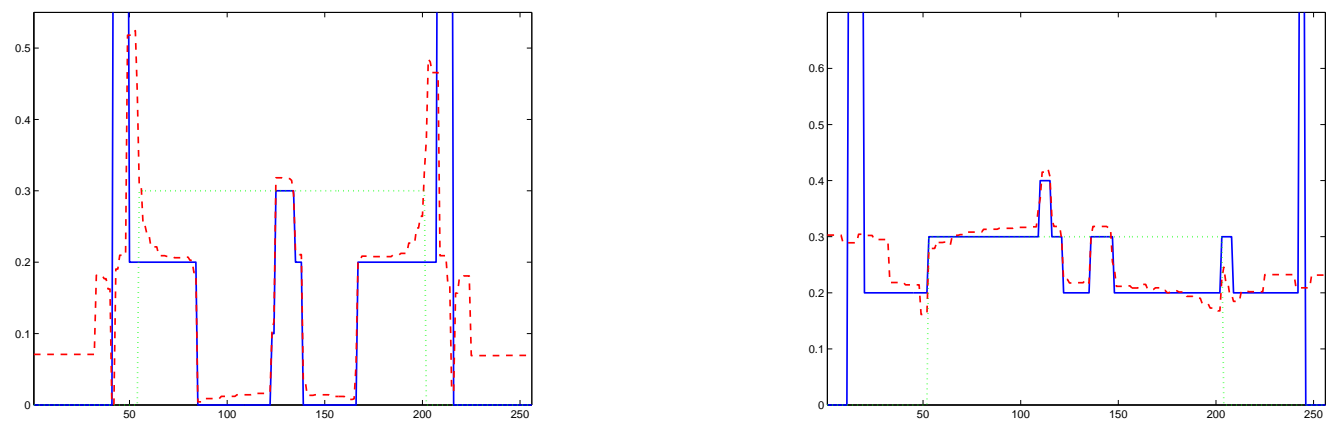

Figure 6 - Profiles along the white lines in Figure 5; true (blue, solid), reconstructed (red, dashed), region of interest (green, dotted).

justifies convergence of our algorithm, at least for continuous data. Numerical tests on phantoms demonstrate that our method works well, and that it is indeed beneficial to allow also small details outside the ROI to be reconstructed. First observations regarding the influence of the weights and the regularization parameter indicate the existence of an optimal pairing. However, parameter and weight choice rules are topic of future work.

\section{REFERENCES}

[1] S. Anzengruber and R. Ramlau. Morozovs discrepancy principle for Tikhonov-type functionals with nonlinear operators. Inverse Problems, 26:025001, 2010.

[2] S. W. Anzengruber, B. Hofmann, and R. Ramlau. On the interplay of basis smoothness and specific range conditions occurring in sparsity regularization. Inverse Problems, 29:125002, 2013.

[3] S. W. Anzengruber and R. Ramlau. Convergence rates for morozovs discrepancy principle using variational inequalities. Inverse Problems, 27:105007, 2011.

[4] T. Bonesky, K. Bredies, D. A. Lorenz, and P. Maass. A generalized conditional gradient method for nonlinear operator equations with sparsity constraints. Inverse Problems, 23(5):2041-2058, 2007.

[5] T. Bonesky, S. Dahlke, P. Maass, and T. Raasch. Adaptive wavelet methods and sparsity reconstruction for inverse heat conduction problems. Adv. Comput. Math., 33(4):385-411, Nov. 2010.

[6] K. Bredies. An iterative thresholding-like algorithm for inverse problems with sparsity constraints in Banach space. J. Inverse Ill-Posed Probl., 17(1):19-26, 2009.

[7] K. Bredies and D. A. Lorenz. Linear convergence of iterative soft-thresholding. J. Fourier Analysis and Applications, 14(5-6):813837, 2008.

[8] M. Burger, J. Flemming, and B. Hofmann. Convergence rates in $\ell_{1}$-regularization if the sparsity assumption fails. Inverse Problems, 29:025013, 2013.

[9] C. Chaux, P. L. Combettes, J.-C. Pesquet, and V. R. Wajs. A variational formulation for frame-based inverse problems. Inverse Problems, 23(4):1495, 2007. 
[10] C. K. Chui. Wavelets: A Mathematical Tool for Signal Analysis. Math. Model. Comput. 1, SIAM, Philadephia, 1997.

[11] A. M. Cormack. Representation of a function by its line integrals with some radiological applications. J. Appl. Physics, 34:2722-2727, 1963.

[12] M. Courdurier, F. Noo, M. Defrise, , and H. Kudo. Solving the interior problem of computed tomography using a priori knowledge. Inverse Problems, 24:065001 (27pp), 2008.

[13] I. Daubechies. Ten lectures on wavelets. CBMS-NSF Regional Conference Series in Applied Mathematics. 61. Philadelphia, PA: SIAM, Society for Industrial and Applied Mathematics. xix, 357 p. , 1992.

[14] I. Daubechies, M. Defrise, and C. De Mol. An iterative thresholding algorithm for linear inverse problems with a sparsity constraint. Comm. Pure Appl. Math., pages 1413-1457, 2004.

[15] I. Daubechies, M. Fornasier, and I. Loris. Accelerated Projected Gradient Method for Linear Inverse Problems with Sparsity Constraints. Journal of Fourier Analysis and Applications, 14(5 - 6), 2008.

[16] A. Delaney and Y. Bresler. Multiresolution Tomographic Reconstruction Using Wavelets. IEEE Trans. Image Processing, 6:799-814, June 1995.

[17] J. DeStefano and T. Olsen. Wavelet localization of the Radon transform in even dimensions. IEEE Trans. Signal Proc., 42:2055-2067, 1994.

[18] D. L. Donoho. Nonlinear solution of linear inverse problems by wavelet-vaguelette decomposition. $A p$ plied and Computational Harmonic Analysis, 2:101-126, 1992.

[19] D. L. Donoho. Compressed sensing. IEEE Trans. Inform. Theory, 52:1289-1306, 2006.

[20] H. W. Engl, M. Hanke, and A. Neubauer. Regularization of Inverse Problems. Kluwer Academic Publishers, Dortrecht, 1996.

[21] A. Faridani, D. Finch, E. L. Ritman, and K. T. Smith. Local tomography, II. SIAM J. Appl. Math., 57:1095-1127, 1997.

[22] A. Faridani, E. L. Ritman, and K. T. Smith. Local tomography. SIAM J. Appl. Math., 52:459-484, 1992.

[23] M. A. T. Figueiredo, R. D. Nowak, and S. J. Wright. Gradient projection for sparse reconstruction: Application to compressed sensing and other inverse problems. Technical report, IEEE Journal of Selected Topics in Signal Processing, 2007.

[24] M. Fornasier. Domain decomposition methods for linear inverse problems with sparsity constraints. Inverse Problems, 23(6):2505, 2007.

[25] D. Gerth and R. Ramlau. A stochastic convergence analysis for Tikhonov regularization with sparsity constraints. Inverse Problems, 30:055009, 2014.

[26] M. Grasmair. Well-posedness and convergence rates for sparse regularization with sublinear $\ell_{q}$ penalty term. Inverse Problems Imaging, 3:0383387, 2009.

[27] M. Grasmair, M. Haltmeier, and O. Scherzer. Sparse regularization with $\ell_{q}$ penalty term. Inverse Problems, 24(5):055020, 2008.

[28] V. Guillemin and S. Sternberg. Geometric Asymptotics. American Mathematical Society, Providence, RI, 1977.

[29] K. Hämäläinen, A. Kallonen, V. Kolehmainen, M. Lassas, K. Niinimäki, and S. Siltanen. Sparse tomography. SIAM J. Sci. Comp., 35(3):B644-B665, 2013.

[30] W. M. Han, H. Yu, and G. Wang. A total variation minimization theorem for compressed sensing-based tomography. International Journal of Biomedical Imaging, page 125871, 2009.

[31] S. Helgason. Integral geometry and Radon transforms. Springer, New York, 2011.

[32] B. Jin, T. Khan, and P. Maass. A reconstruction algorithm for electrical impedance tomography based on sparsity regularization. Int. J. Numer. Meth. Engng., 89:337-353, 2012.

[33] B. Jin and P. Maass. Sparsity regularization for parameter identification problems. Inverse Problems, 28:123001, 2012.

[34] M. Kalke and S. Siltanen. Adaptive frequency-domain regularization for sparse-data tomography. Inverse Problems in Science and Engineering, 21(7), 2006.

[35] A. I. Katsevich and A. G. Ramm. Pseudolocal tomography. SIAM J. Appl. Math., 56:167-191, 1996.

[36] F. Keinert. Wavelets and Multiwavelets. Chapman and Hall, London, 2004.

[37] E. Klann. A Mumford-Shah-Like Method for Limited Data Tomography with an Application to Electron Tomography. SIAM J. Imaging Sciences, 4(4):1029-1048, 2011. 
[38] E. Klann, M. Kuhn, D. A. Lorenz, P. Maass, and H. Thiele. Shrinkage versus deconvolution. Inverse Problems, 23(5):2231, 2007.

[39] E. Klann, R. Ramlau, and W. Ring. A Mumford-Shah level-set approach for the inversion and segmentation of SPECT/CT data. Inverse Problems and Imaging, 5(1):137-166, 2011.

[40] V. Kolehmainen, M. Lassas, K. Niinimäki, and S. Siltanen. Sparsity-promoting Bayesian inversion. Inverse Problems, 28(2):025005, 2012.

[41] D. Lorenz. Convergence rates and source conditions for Tikhonov regularization with sparsity constraints. J.Inverse Ill-Posed Problems, 2008.

[42] A. Louis. Inverse und schlecht gestellte Probleme. Teubner Verlag, Stuttgart, 1988.

[43] A. K. Louis, P. Maass, and A. Rieder. Wavelets. Theory and applications. Pure and Applied Mathematics. A Wiley-Interscience Series of Texts, Monographs \& Tracts. Chichester: John Wiley \& Sons. xvii, 324 p., 1997.

[44] A. K. Louis and A. Rieder. Incomplete data problems in X-ray computerized tomography II. Truncated projections and region-of-interest tomography. Numerische Mathematik, 56:371-383, 1986.

[45] P. Maaß. The interior Radon transform. SIAM J. Appl. Math., 52:710-724, 1992.

[46] W. Madych. Tomography, Approximate Reconstruction, and Continuous Wavelet Transforms. Appl. Comp. Harm. Anal., 7:54-100, 1999.

[47] F. Natterer. The Mathematics of Computerized Tomography, volume 32 of Classics in Applied Mathematics. Society for Industrial and Applied Mathematics (SIAM), Philadelphia, PA, 2001. Reprint of the 1986 original.

[48] K. Niinimäki, S. Siltanen, and V. Kolehmainen. Bayesian multiresolution method for local tomography in dental X-ray imaging. Physics in Medicine and Biology, 52:6663-6678, 2007.

[49] E. T. Quinto. Singularities of the X-ray transform and limited data tomography in $\mathbb{R}^{2}$ and $\mathbb{R}^{3}$. SIAM J. Math. Anal., 24:1215-1225, 1993.

[50] R. Ramlau. Regularization properties of Tikhonov regularization with sparsity constraints. ETNA. Electronic Transactions on Numerical Analysis [electronic only], 30:54-74, 2008.

[51] R. Ramlau and E. Resmerita. Convergence rates for regularization with sparsity constraints. Electron. Trans. Numer. Anal., 37:87104, 2010.

[52] R. Ramlau and G. Teschke. Sparse recovery in inverse problems. In M. Fornasier, editor, Theoretical Foundations and Numerical Methods for Sparse Recovery, volume 9 of Radon Series on Computational and Applied Mathematics. De Gruyter, 1993.

[53] R. Ramlau and G. Teschke. A Tikhonov-based projection iteration for nonlinear ill-posed problems with sparsity constraints. Numerische Mathematik, 104(2):177-203, Aug. 2006.

[54] R. Ramlau and C. A. Zarzer. On the minimization of a Tikhonov functional with a non-convex sparsity constraint. Electron. Trans. Numer. Anal., 39:476507, 2012.

[55] M. Rantala, S. Vanska, S. Järvenpää, M. Kalke, M. Lassas, J. Moberg, and S. Siltanen. Wavelet-based reconstruction for limited-angle x-ray tomography. IEEE Trans. Med. Imaging, 25(2):210-217, 2006.

[56] F. Rashid-Farrokhi, K. Liu, C. A. Berenstein, and D. Walnut. Wavelet-based multiresolution local tomography. IEEE Trans. Image Processing, 6:1412-1430, 1997.

[57] B. Sahiner and A. Yagle. Limited angle tomography using wavelets. In Nuclear Science Symposium and Medical Imaging Conference, volume 3 of IEEE Conference Record, pages 1912 - 1916. IEEE, 1993.

[58] B. Sahiner and A. E. Yagle. Region-of-interest tomography using exponential radial sampling. IEEE Transactions on Image Processing, 4(8):1120-1127, 1995.

[59] J. Terry, B. Tsui, J. Perry, J. Hendricks, and G. Gullberg. The design of a mathematical phantom of the upper human torso for use in 3-d spect imaging research. In: Proc. 1990 Fall Meeting Biomed. Eng. Soc., Blacksburg, VA, New York University Press., pages 1467-1474, 1990.

[60] G. Teschke and C. Borries. Accelerated projected steepest descent method for nonlinear inverse problems with sparsity constraints. Inverse Problems, 26(2):025007, 2010.

[61] H. Triebel. Function Spaces and Wavelets on Domains. EMS Tracts Math. 7, European Mathematical Society, Zürich, 2008.

[62] E. Vainberg, I. A. Kazak, and V. P. Kurozaev. Reconstruction of the internal three-dimensional structure of objects based on real-time integral projections. Soviet Journal of Nondestructive Testing, 17:415-423, 1981. 
[63] M. Wernick and J. e. Aarsvold. Emission Tomography. The Fundamentals of PET and SPECT. Elsevier Academic Press, 2004.

[64] J. Yang, H. Yu, M. Jiang, and G. Wang. High-order total variation minimization for interior tomography. Inverse Problems, 26(3):035013, 2010.

[65] Y. Ye, H. Yu, and G. Wang. Exact interior reconstruction from truncated limited-angle projection data. International Journal of Biomedical Imaging, 6, 2008.

[66] H. Yu and G. Wang. Compressed sensing based interior tomography. Physics in Medicine and Biology, 54:2791-2805, 2009.

[67] H. Yu, J. Yang, M. Jiang, and G. Wang. Supplemental analysis on compressed sensing based interior Tomography. Physics in Medicine and Biology, 54:N425-N432, 2009.

[68] C. A. Zarzer. On Tikhonov regularization with non-convex sparsity constraints. Inverse Problems, 25:02006, 2009.

ESTHER KLANN, JOHANNES KEPLER UNIVERSITY LINZ, ALTENBERGER STR. 69, 4040 LINZ, AUSTRIA

E-mail address: ESTHER.KLANN@JKU.AT

ERIC TODD QUINTO, DEPARTMENT OF MATHEMATICS, TUFTS UNIVERSITY, 503 BOSTON AV., MEDFORD, MA 02155, USA

E-mail address: TODD.QUINTO@TUFTS.EDU

RONNY RAMLAU, JOHANNES KEPLER UNIVERSITY LINZ, ALTENBERGER STR. 69, 4040 LINZ, AUSTRIA; AND RADON INSTITUTE FOR COMPUTATIONAL AND APPLIED MATHEMATICS, ALTENBERGER STR. 69, 4040 LINZ, AUSTRIA

E-mail address: RONNY.RAMLAU@JKU.AT 